News Watch

\title{
NEW TRENDS IN INTERNATIONAL PUBLIC HEALTH LAUNCH OF A NEW DIPLOMA, MSc \& PhD PROGRAMME IN RESEARCH \& DEVELOPMENT OF PRODUCTS TO MEET PUBLIC HEALTH NEEDS
}

Kenji Hirayama, M.D., Ph.D.

Editor-in-Chief

Tropical Medicine and Health

Based on the idea that more human resources are needed in the drug/vaccine/diagnostics research and development field in the developing countries where new products are required to improve their unique health situation different from developed countries, a new educational programme has been launched in Japan. Here, I would like to show briefly its philosophy, strategy and contents. This programme has not been completed, however, we have started a core diploma course that is consisted of seven modules that cover all the steps to develop a new product from a very basic discovery. This course has been made possible by a synergistic cooperation between several good persons from various institutions. Their names and institutions are as follows, Win Gutterrage, Janis Lazdins, Juntra Karbwang (World Health Organisation, Switzerland), JinHong $\mathrm{Hu}$ (Second Military Medical University, China), Kesara NaBangchang (Thammasat University, Thailand), Chitr Sitthiamorn (Chulalongkorn University, Thailand), Kiichiro Tsutani (University of Tokyo, Japan), Ivan Valez (University of Antioquia, Colombia), and Kenji Hirayama (Nagasaki University, Japan).

I would like to acknowledge Kazuhiko Mori, Center for Product Evaluation, Pharmaceutical and Medical Devices, PMDA, Japan, Kihito Takahashi, Japanese Association of Pharmaceutical Medicine (JAPHMED), Masaru Iwasaki, GlaxoSmithKline, Tokyo, Japan, Masakatsu Shibasaki, (The University of Tokyo, and The Pharmaceutical Society of Japan, PSJ), Hiroshi Saitoh (President, Nagasaki University) for their great contribution to the diploma course.

Website: http://www.tm.nagasaki-u.ac.jp/hiraken /information/deploma/diploma_top_frame.html

\section{BACKGROUND:}

The development of new drugs, vaccines and diagnostics is complex, requiring many different skills. Each individual involved in a part of product $R \& D$ must be aware of the process overall and be able to relate their activities to it and to the needs of the other participating scientists and clinicians. Research scientists seek the discovery and confirmation of new knowledge by initiating or creating a hypothesis and then transforming it first into a theory and later into a new discovery. Product developers turn such discoveries into full-grown products which address public health needs through a long and quite different processes. Generally, research and development are two different disciplines. People working in these two areas do not think alike; they have different cultures. Often they work in isolation from each other, so they do not understand each other well. However, both disciplines are essential for the development of new drugs, vaccines and diagnostics. Furthermore, discovery of new knowledge is meaningless if it is not translated into new products that meet the needs of the public health.

Currently, there are only few courses in the north that give a good overview of the entire drug discovery and development process such as post-graduate courses at University of Cardiff, ECPM at University of Basel, University of Lyon and may be a few others. In majority of countries around the world, most of the topics related to product $R \& D$ are scattered throughout the various university curricula, including basic science, organic chemistry, immunology, pharmacy, pharmacology, vaccinology and clinical pharmacology. However, everyone involved in research or development must know their responsibilities and able to relate their tasks to all the others which make up the process of product R\&D. The objective of the proposed course is to pull together the various components needed for product $\mathrm{R}$ $\& D$ into a dedicated MSc-PhD course. Discussions between different universities who are interested in this project have taken place on several occasions. Recently, six universities in four countries (Japan, Thailand, China and Colombia) have started working together to draft the content of the curriculum. 
The curriculum is designed to provide basic knowledge of the product $R \& D$ process. It aims to demonstrate that new product discovery and the various development activities such as chemistry, toxicology, clinical investigations and regulatory practices are related as a continuous process, and that one discipline cannot carry out the whole process on its own.

\section{Format of the course:}

This is a joint project involving a number of universities in different countries. During the first five weeks, all students will take a core course on product $\mathrm{R} \& \mathrm{D}$ aimed at giving them an overview of the process. It will be held on a rotational basis in one of the participating universities. During the next ten weeks, students will select for in-depth exploration an area of their special interest, for example drug discovery, toxicology or clinical, and for this period will work in an institution that offers that specific area.

For the last 8 months, students will be attached to a specific institution, pharmaceutical company or biotech to work on a particular project.

The first four months of the course will be mainly lectures and case studies. The remaining 8 months will be mainly practical, including laboratory studies or clinical practice. The course is modular to allow those who already work in the area of product $R \& D$ to attend the appropriate parts of the course. This would provide such personnel with the opportunity of reviewing and discussing the special problems they encountered in their routine, real world work.

At the end of five weeks, students will be awarded a diploma in product research and development from Nagasaki University.

At the end of the first year, the successful students may receive a MSc. (subject to university requirement). Progression to $\mathrm{PhD}$ will depend on the evaluation of their first year performance. Those chosen as $\mathrm{PhD}$ candidates will do their thesis in the area of interest in one of the companies with on -going activities. Each student will receive their degree from the university that they registered for the course.

\section{Contents of the core diploma course}

This part of the course will begin with a general overview of product R\&D (drugs, vaccines and diagnostics), continue with a series of lectures on discovery research, transitioning between research and development, CMC (chemical, manufacturing and control) requirements, toxicology requirements from development to product license, pharmaceutical and analytical development, pharmacokinetics and metabolic studies, clinical studies - phase I-IV trial design and protocols - project planning and management, handling of safety data in development, regulatory requirements, post regulatory clinical studies, patents issues and other aspects of product development i.e. international standards of good practice, ethics in clinical research, DSMB (Data and safety Monitoring Boards), commercial and marketing activities, and public health implementation.

\section{Contents of the course during the next ten weeks:}

The students will focus on the area of their interest. They will attend seminars and lectures in specific area such as discovery, CMC, toxicology, vaccinology, pharmacology, clinical development and regulatory requirements. For this, the student can choose to attend at their registered university (if such course is running during the period) or at another affiliated institution. 


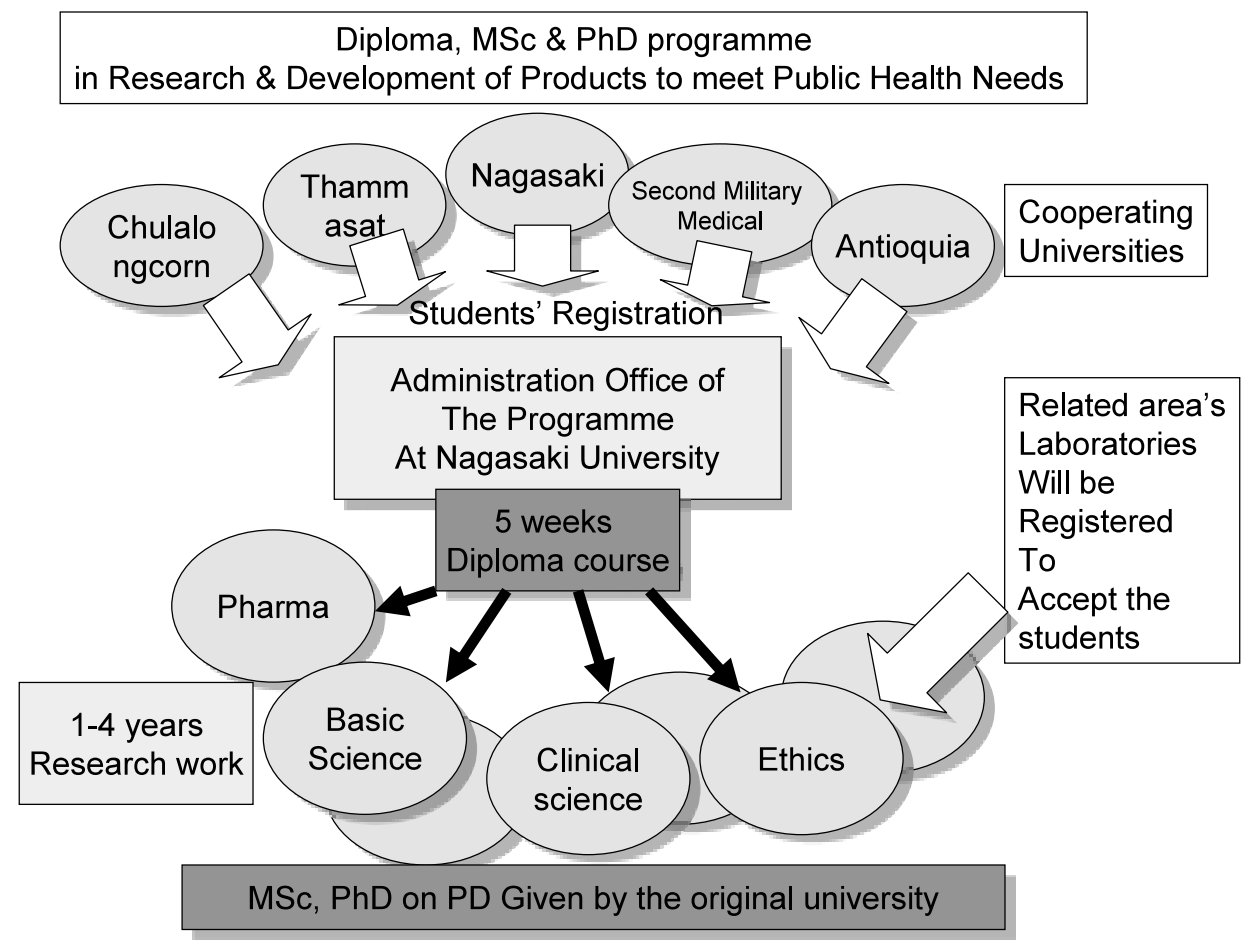

\section{The program of core course in product $R \& D$ (Syllabus)}

\section{Module 1: Course Orientation}

This module provides an over view of the need for a specific drug or vaccine for a disease or condition. The challenge for pharmaceutical research is to analyze a disease or condition to determine its effect on the body. This analysis leads to discovery of drug, vaccine or diagnostic which could then turn into a product. Resource requirements of current development paradigm and the time taken to develop a new product will be discussed.

The following topics will be covered:

$\bigcirc$ Key medical and public health issues, and the need for new products

$\circ$ Discovery research and product development and the different approaches required for each of them

$\circ$ Resource implication for product development

O Stakeholders in Product Research and Development

O Major players

- Large, medium and small pharmaceutical companies

- Academic institutions

- $\mathrm{CRO}$

- Biotechs

- Regulatory

\section{Module 2: Drug Development}

\section{O Discovery Research:}

A comprehensive review of various approaches for new drug discovery from early history of mankind to contemporary techniques such as rational drug design, medicinal chemistry, in silico technology, genomics, proteomics, and pharmacogenomics will be covered. Examples of therapeutic areas derived from these approaches will be provided including lessons learned, pitfalls, and evolution towards a newer and more efficient approach. The importance of having the patents protected in the early stage as well as the strategy to publish (or not publish) the novel findings will be discussed.

The following topics will be covered:

- Historical

- Overview of modern drug discovery

- Drug Targets

- Lead Generation

- Lead Optimization

- Patents and publications

$\bigcirc$ Chemical Development: Chemistry, manufacturing and control (CMC):

The required standards for composition, manufacture process, and controls information of the drug substance and the drug product that can ensure proper identification, quality, purity and strength of the investigating product will be described. 
Following topics will be covered:

- Synthesis of active pharmaceutical ingredient

- Formulation

- Methods for determination of concentrations in various media by means of spectrometric methods, electromechanical methods, HPLC, gas chromatography and biological methods

- Stability for drug substance and drug product

- Development of specification

- Quality assurance/quality control

- Regulatory (with an example of a drug CMC requirement)

- Naming the New Chemical Entity

\section{$\bigcirc$ Preclinical development}

The purpose of the Preclinical studies is to evaluate the pharmacological activity and toxicity of a drug candidate. The contents describe principles of pharmacology and toxicology, including the types of pharmacological and toxicological testing both in vitro and in vivo (animal model) and pharmacokinetics.

The following topics will be covered:

- Pharmacological development

- Principles \& knowledge of methodology

- Pharmacological Tests:

$\checkmark$ in vitro

$\checkmark$ Animal models, selection of suitable model and design

- Toxicology

- Principles of Toxicology

- Toxicological Tests: in vitro \& in vivo: acute, subacute, chronic, special organ toxicology, reproduction toxicology, teratogenicity, mutagenicity, carcinogenicity studies

- Scheduling of toxicological studies in the development plan, the registration requirements, human \& animal pharmacology, the proposed clinical application and the forms of administration.

- Continuous monitoring of the correlation between new toxicological findings and the unwanted events observed in humans up till now.

- Pre-clinical Pharmacokinetics

- Principles of Pharmacokinetics

$\checkmark$ ADME Processes

$\checkmark$ Pharmacokinetic Data Analysis \& Pharmacokinetic Parameters

- Transferability of the pharmacokinetic findings in animals to humans

- Investigating toxicological problems - practices and pitfalls

- Evaluation of viability (risk and benefit) for fur- ther development (case study)

\section{$\circ$ Clinical development}

The overview of clinical development will be presented, including the assessment of pre-clinical information package and clinical development plan. The important of human pharmacokinetics and pharmacodynamics in drug development will be discussed. Description of different clinical trial phases will be presented with examples. Different trial designs suitable for each type of studies will be illustrated, sample size calculation, statistical analysis plan and issues encountered during clinical development will be emphasized. An example of statistical analysis report will be demonstrated. Clinical data management methodology will be introduced. The regulatory requirements for clinical development and process of regulatory submission will be discussed.

Following topics will be covered:

- Overview

- Assessment of pre-clinical information

- Clinical development plan

- Application of pharmacokinetics and pharmacodynamics in drug development

- Dose selection and regimen

- Clinical trials

- The various investigational phases of clinical research (Phases I-IV)

$\checkmark$ Human pharmacokinetics and pharmacogenetics

- Definition and significance of pharmacokinetic parameters ( absorption , bioavailability, binding to proteins, distribution, clearance, elimination half life, AUC)

- Special human-pharmacokinetic studies e. g. bioavailability studies of multiple-dose, interaction studies, pregnancy, liver disease $e t c$.

$\checkmark$ Therapeutic exploratory

$\checkmark$ Therapeutic confirmatory

$\checkmark$ Therapeutic use

$\checkmark$ Safety monitoring and reporting in clinical trials

- Basic principles and evaluation of investigational results

(Phase-I and early Phase-II), with a view to further development

- Basic principles for decisions regarding further development or Discontinuation of a development project

- Study design 
- possible study designs taking into account ethical aspects, indication, controls, patient population, location of the trial centers

- Trial design (parallel group design, cross over design, factorial design)

- Design techniques to avoid bias (blinding, randomization)

- Multi centers trials

- Type of comparison

- Group sequential designs

- Outcome measurements

- Statistical considerations

- biostatistics in the planning phase (estimate of number of cases, randomization, statistical models, definition of end-points, planning of the subsequent evaluation)

- Statistical analysis plan

- Analysis sets: full analysis set, per protocol set, missing values and outliers

- Data transformation

- Method of statistical analysis (estimation, confidence intervals, hypothesis testing, evaluation of safety and tolerability

- Statistical analysis report

- Introduction to Clinical Data Management

- Regulatory aspects of clinical development

\section{Module 3: Vaccine Development}

Rational: World eradication of small pox clearly showed that vaccine is a powerful tool to control the disease. First of all, we will discuss about the reasons why some vaccine developments were successful whereas some were not. Then we will discuss what types of vaccine or vaccine development are ideal to control the major diseases such as infectious diseases, cancer, autoimmune diseases etc.

\section{$\bigcirc$ Discovery Research - Vaccines}

Vaccine effect is dependent on the acquired immunity that directed for the elimination of non-self including microorganisms, parasites, and cancer cells induced by the immunization of some appropriate antigens. What is the most effective acquired immunity against the disease? How do you select good antigens for such an effective vaccine development? And how do you make that antigen(s) more effective? We will see the most advanced knowledge and technology that will facilitate vaccine discovery research.

A historical review and overview of modern vaccine discovery will be presented. Various methods for antigen screening such as bioinformatics, protein chemistry, recombinant antigens, high-throughput, cell free recombinant system will be discussed. The evaluation of candidate antigens and selection of candidates for development will be described. Alternatives to using antigens will be presented. Examples of antigens derived from these approaches will be provided including lessons learnt, pitfalls, and evolution towards a newer and more efficient approach. Different adjuvant will be explored and discussed. The importance of having the patents protected in the early stage as well as the strategy to publish the novel findings will be discussed.

- Historical

- Overview of modern vaccine discovery

- Understanding the basic immunology of the disease:

Acquired immunity, Protective immunity, antigen, immunogenic

- Biological Targets and Vaccine Candidates identification

- Infectious disease, Cancer therapy, Autoimmune,

- Screening for antigens

- Bioinformatics, Protein chemistry, recombinant antigens, high-throughput method including cell free recombinant system

- Evaluating antigens

- In vitro and in vivo tests including animal model. Restriction for using animal model, genetically engineered animals

- Adjuvant

- Type of the immune response provoked, new type of adjuvant, recombinant cytokine as adjuvant

- Alternatives to antigens

- DNA vaccine, Live or attenuated pathogen

- Selection of development candidate and back-ups

- Efficacy, toxicity, route if immunization, price, stability, cold chain,

- Patents and publications

\section{$\bigcirc$ Antigen development}

After or during identification of vaccine antigens, it will be necessary to prepare appropriate amount and quality of antigens for further study. Process of scale-up, manufacture and control of the antigen will be demonstrated. Formulation of the vaccine antigen according to the required standard will be discussed.

- Scale-up, manufacture and control:

- Types of large scale production of antigen, GLP and GMP

- Synthesis of antigen

- Peptide, recombinant protein, DNA vaccine, recombinant $\mathrm{BCG}$ or organism, live or attenuated organism 
- Synthesis of adjuvant

- Mixture type or recombinant type

- Formulation

- Soluble or suspension, Route, frequency, interval, number of dose, with or without adjuvant, mucosal immunization (aerosol, oral, nasal, inhalation, food), instability

- Quality assurance/quality control,

- Regulatory

\section{P Preclinical development}

Preclinical Safety and immunogenicity assessment for vaccine development is performed by using animal model. There are two major check points, one is injection sites and the other systemic effect such as hypersensitivity and autoimmunity. Overview of the preclinical research will be demonstrated by using several typical experiences for vaccine development. Methods for the safety assessment and immunogenicity will be demonstrated. There will be a special session to demonstrate the classical and novel concept of animal model.

\section{- Safety assessment}

Toxicity test for animal: regional complications, systemic toxicity such as fever, anaphylactic shock,

- Immunogenicity assessment

- Animal model used in pre-clinical studies

- The use of humanized animal model

\section{$\circ$ Clinical Development}

Clinical development of vaccine is somehow different from that of drug especially in the evaluation process and ethical aspects. The participants in vaccine trial are usually healthy volunteers and the sample size is normally greater than drug trial. It also takes longer duration to demonstrate the efficacy e.g. it needs a naturally infection to demonstrate the effect as challenging infection is not ethically acceptable. Administration of vaccine requires more complex procedures than drug trials, for example it requires cold chain, injection instruments, health workers, etc.

The assessment of pre-clinical information to proceed to human will be emphasized. In the Clinical trial section, the various investigational phases of clinical research (Phases IIV) will be demonstrated. Basic principles and evaluation of investigation, development project, Study design, Statistical considerations, Data transformation Clinical Data Management, regulatory

- Overview

- Assessment of pre-clinical information

- Clinical development plan

- Application of immunogenicity for vaccine development

- Dose selection and regimen

- Clinical trial

- The various investigational phases of clinical research (Phases I-IV)

$\checkmark$ Human immunogenicity and evaluation of efficacy

$\checkmark$ Confirmatory Studies

$\checkmark$ Vaccine use

$\checkmark$ Safety monitoring and reporting in clinical trials

- Basic principles and evaluation of investigational results

(Phase-I and early Phase-II), with a view to further development

- Basic principles for decisions regarding further development or discontinuation of a development project

- Study design

- possible study designs taking into account ethical aspects, indication, controls, population, location of the trial centers

- Trial design (parallel group design, longitudinal design, factorial design, group sequential designs

- Design techniques to avoid bias (blinding, randomization)

- Multi centers trials

- Type of comparison

- Outcome measurements

- Statistical considerations

- biostatistics in the planning phase (estimate of number of cases, randomization, statistical models, definition of end-points, planning of the subsequent evaluation)

- Statistical analysis plan

- Analysis sets: full analysis set, per protocol set, missing values and outliers

- Data transformation

- Method of statistical analysis (estimation, confidence intervals, hypothesis testing, evaluation of safety and tolerability

- Statistical analysis report

- Introduction to Clinical Data Management

- Regulatory

\section{Module 4: Diagnostic Development}

Diagnostic tools in combination with therapeutic or preventive medical care are important to develop for public health purpose. Without good diagnostic method, it would be impossible to evaluate the disease burden in the community, to 
treat the patients and to protect the society against the disease.

Practical approach towards the development of really effective diagnostic tools for public health will be demonstrated and discussed. In the overview, several excellent examples will be shown. Discovery session will show three steps for the discovery, Necessity assessment, technology selection, prototype production. Evaluation of clinical applicability such as Sensitivity and specificity will be discussed. Detailed protocol for the Clinical development will be demonstrated.

- Overview

- Discovery and Development of diagnostic tools

- Necessity assessment, Principles and technology selection, prototype production and assessment.

- Identify preliminary diagnostic test

- Validation of clinical potential

- Identification of new targets using genomics and protein and cellular studies

- Development of potential technology platform

- Principles of diagnostic methods antibody detection, antigen detection, biological parameters including DNA, RNA, enzymes, proteins

- Define product specifications

- Feasibility assessment

- Scale-up, manufacture and control:

- Practical Application:

Development of kits, necessary equipments, electricity, technician, budget

- Quality assurance/quality control: Evaluation of the efficacy after application

- Clinical Development:

- Validate prototype

- Manufacture pilot lot

- Initiate clinical trials

- Supply chain logistics and production

- Implementation

- Statistical considerations

- Regulatory matters

\section{Module 5: Standards in Clinical Research and Develop- ment}

Regulations and guidelines vary from one country to another. These regulations and guidelines dictate on how to develop the product in each country. The product developer must meet all the requirements and expectations of the regulatory authorities as efficiently as possible. The module describes the guidelines and regulatory requirements in various countries.
- Good Manufacturing Practice:

- Good Laboratory Practice:

- Good Clinical Practice:

- ICH

- WHO

- Ethics Codes and Guidelines:

- Declaration of Helsinki

- CIOMS

- Belmont Report

- WHO Operational Guidelines for Ethics

Committees that Review Biomedical Research

- Principles of Research Ethics Autonomy, Beneficence, Justice

- Research Methodologies and Ethical Issues

Biomedical Research including traditional medicine

In Various Types of Health Research Genetic Research \& Stored Samples

- Ethics Committees

- Data and Safety Monitoring Boards

- Clinical Data Management

- Data protection aspects

- Clinical study monitoring

- Audits and inspections

- US FDA Guidelines and regulation

- EMEA Guidelines and regulations

- Japan Guidelines and regulations

- China Guidelines and regulations

- Thailand Guidelines and regulations

- Colombia Guidelines and regulations

\section{Module 6: Clinical Data Management}

Project planning and management at every stage of development will be described. Developmental objectives, crucial milestones, concise detailed analysis of product and roadmap to market will be demonstrated, including Patent process. Regulatory strategies and strategies for dealing with potential roadblocks and hurdles in the product development process will be discussed. The plan will include a lay out of an accurate and realistic budgets and timelines throughout the project development. A practical workshop on project planning and management will be included. Following topics will be covered:

- Project planning and management, including practical session

- Data acquisition

- Data Privacy

- Data Capture Principles

- CRF Design \& Completion Guidelines

- Electronic Data Capture

- Database 


\author{
- Data Storage \\ - Database Validation \\ - Database Programming and Standards \\ - Data \\ - Data Entry \\ - Data Processing: Validation (Edit Check \\ Specification) \\ - Laboratory \& External Data \\ - Dictionary Management \\ - Adverse Events \\ - Drug \\ - Reporting \\ - Safety Data Management and Reporting
}

\section{Module 7: Activities after Registration}

Objective:

a. to identify stakeholders to be involved in post regulatory activities, their functions and roles in bringing the products to solve the intended public health problems.

b. describe the policy instruments to bring the products to the intended beneficiaries.

c. develop strategies for public and private partnership to encourage

- Research and development in areas of need and

- Advocate the public sector to allocate funds to allocate funds to bring products to the intended beneficiaries.

d. develop evidence based actionable message, identify resources requirement to scale up the products for use in the health care system (diagnostic tests, vaccines and drugs) as well as describe strategies to mobilize these resources.

e. describe mechanisms and strategies for post marketing product vigilance for product quality, post marketing efficacy and side effects.

f. Identify the human resource capacity strengthening needs and strategies to fulfil those needs such as through best practice health services research using "unqualified" personnel and training of the trainers.

\section{○ Stakeholders to be involved in making product de-} velopment work for the intended beneficiaries

Stakeholders in health care systems are important in making development products work for poor people and intended beneficiaries. These include the policy makers, the system managers, directors of facilities, the practitioners and the intended beneficiaries. Each of these stakeholders has unique responsibility, roles and functions. The roles and functions have to be coordinated to make the system make the prod- ucts work. The unique roles, functions and the tools to coordinate the stakeholders to make the product work will be described with specific examples for HIV/TB care and addressing poverty gender based inequalities and how to deal with them. Specific stakeholders to be discussed in details are:

- Public policy

- System policy

- Facility Policy

- Practice Policy

- Empowerment of public

\section{$\bigcirc$ Policy Instrument}

Social factors to a large extent shape the success for failure of bring innovative products to benefit the intended beneficiaries. Policy instruments are needed to deal with the complexity of social impediments to health and diseases. The use of the policy instruments will need the right understanding of diseases and their proximate and distant determinants. The right understanding can give insight to: a) targeting the products; b) development rules and regulation to procure and provide the products to the intended beneficiaries; c) allocation of resource to finance the purchase of the products to target beneficiaries and d) development of services either at the public or private sectors where appropriate. Specific instruments to be discussed in details are:

- Public health need and vulnerable groups

- Targeting

- Rule regulation (financing, guidelines)

- Resources allocation

- Service planning: primary, secondary, tertiary care

\section{- Public Private Partnership (3 hours)}

The purpose of partnership between the public and private sector is to promote the interface between product development and their use in clinical and systems settings. Partnership can have an effect of the overall priorities and successes of product development. A good partnership will strengthen the credibility of the relationship between the public and private sector over the long term. There are several possible reasons to develop a public and private partnership. The most important one should be the need to achieve a task, which is not achievable if each of the partners works independently. Typically, these activities help control a 'neglected' disease or condition in developing countries such as through development or distribution of a drug, diagnostics, vaccines, contraceptive and other products. In general, there are two types of partnership:

1) those that want to tackle a problem in a more efficient way; and 
2) those that are created to tackle what is conceived as intractable problems such as the development of a malaria vaccine. These partnerships want to find new ways of tackling the problems because the world does not yet know how to do it. Since the cost of product development can be high, economic consideration to promote an interface between development/clinical use and approval, post marketing must be in place. Both the "push" and "pull" mechanisms will be described. The "push" mechanism guide the research and development initiatives, while the "pull" mechanism ensure that the public sector will guarantee allocation of funds to purchase products for the intended beneficiaries once they are available.

- Public-private partnership

- Function and structure of partnerships

- Good characteristic of partnerships

- Monitoring partnerships

- Examples of partnerships for product research and development

\section{$\bigcirc$ Improving the quality use of new products in health systems}

Evidence for efficacy and safety of the products must be interpreted for potential users of the products to enhance quality use for intended beneficiaries. The potential users include policy makers, practitioners, patients and public, including the media. The interpretations have to be transformed into evidence based actionable products relevant for each of potential users. Other strategies such as the "triangle that moves the mountain" and the "academic NGO" movement of the University of Ottawa can be used to develop strategies to link evidence based actionable message to potential users.

\section{- Diagnosis}

- Characteristics of tests and resource needed to implement the test in health system.

- Access to diagnostic services and case finding for poverty and gender-based inequalities

- Balancing public protection and stigmatization and denial (TD/HIV)

- Vaccine:

- Characteristics of Vaccine and resource needed to implement the test in health system.

- Coverage and herd immunity

- Post vaccination exposure and risk activities

- DRUG.

- Indications, contraindications, use and resource needed to implement the test in health system.

- Compliance of provider

- Compliance of subjects

- Measures to improve compliance

\section{$\bigcirc$ Post marketing product vigilance}

New products, such as drugs, vaccines and diagnostic tests have both benefits and side effects, some of which might not be apparent until the products have been used over a long period of time. Therefore, a system must be developed to measure the benefits as well as risks. Benefits need to be weighed against the occurrence of adverse events. A risk/ benefit analysis of the products must be evaluated using standardized tools and procedures. The importance of guidance for standardization of terminology, data collection, verification and presentation of efficacy and adverse reaction reports will be emphasized and discussed.

Possible topics include:

- The definition of pharmacovigilance.

- The scope, instruments/tools, and processes needed for Pharmacovigilance of medicinal products for human use.

- Systems for standardization of pharmacovigilance reporting and exchange of pharmacovigilance information and subsequent appropriate actions.

- Administrative and legislative information relevant to medicinal products for human use.

- The mechanism for reviewing and updating legislative and technical areas for general use.

Capacity for optimal delivery of new product: Training and Health services research

It is important that countries have the capacity to identify, innovate and adapt new products to its own need and constraints in order to address their unique burden of illnesses including the burden of tropical diseases. At times, health service research might be carried out to document the possibility of using products via "unqualified personnel" through training to ensure best practice (such as the use of nurses for provide contraceptive services). Policy formulation, implementation and evaluation must be in place. Most developing countries do not have capacity to formulate policy identify, innovate and adapt new products to relevant to their problems. Vaccines against ROTA virus, which was not approved in the US due to rate intussusceptions might have be very beneficial in developing countries in preventing burden of illnesses from ROTA virus diarrhea over the incidence of complications because the incidence of ROTA virus diarrhea is high. Likewise, capacities for the development of treatment guidelines and their financing, and optimal facility planning for new products are needed to optimally distribute and use the new products for the intended beneficiaries. Individual practitioners also need skills to search for the best evidence about the use of products within the constraints of their health systems. Various models of international collaboration for capacity strengthening are available 
such as the Thai Golden Jubilee grant. The topics to be considered and discussed include:

- Policy formulation, implementation and evaluation

- Guidelines \& Finance (insurance)

- Optimal facility planning and program manage- ment.

- Evidence based search for best information for practitioners, subjects

- Model for capacity strengthening through international collaboration.

Agenda of the diploma course of 2006

\author{
Diploma Course on Research \& Development of Products \\ to Meet Public Health Needs \\ Sponsored by Nagasaki University \\ in cooperation with Thammasat University, Chulalongkorn University, \\ China Second Military Medical University, Antioquia University \\ and The Graduate School of Pharmaceutical Sciences of The University of Tokyo \\ in collaboration with WHO and The Pharmaceutical Society of Japan (PSJ) \\ Nagasaki University, Japan \\ October 2 - November 8, 2006
}

\title{
Tentative agenda
}

\section{Module 1 Course Orientation}

\section{October 2, 2006 Monday}

\section{0-0915 Welcome address}

President, Prof Dr. Hiroshi Saitoh, Nagasaki

University, Japan

0915-0945 Objective of the course

Professor Dr. Kenji Hirayama, Director of the course, Nagasaki University, Japan

$0945-1000$

$1000-1030$

Introductions of participants

1030-1200 Key medical and public health issues, and the need for new products

Dr. Janis Lazdins, WHO/TDR, Geneva

1200-1300 Lunch

1300-1400 Discovery research and product development and the different approaches required for each of them

Dr. Janis Lazdins, WHO/TDR, Geneva

1400-1500 Stakeholders in Product Research and Development

- Large, medium and small pharmaceutical companies

- Academic institutions

- Clinical Research Organization

- Biotech

- Regulatory

Prof. Dr. Eiji Uchida, Showa University, To- kyo, Japan

1500-1530 Tea break

1530-1600 Stakeholders in Product Research and Development

1600-1630 Q\&A

Module 2 Drug Development Drug Discovery

October 3, 2006 Tuesday

0900-11.00 History and overview of modern drug discovery

Mr Nobuhiro Noro, GSK, Japan

1100-1130 Tea Break

1130-1230 From drug target to drug lead Mr Nobuhiro Noro, GSK, Japan

1230-1330 Lunch

1330-1430 Drug targets identification and validation in malaria

$T B A$

1430-1530 Drug targets identification and validation in TB

Assoc. Prof. Dr. Prasit Palithapolkarnpim, BIOTEC, Thailand

1530-1600 Tea break

1600-1700 Drug targets identification and validation in cardiovascular diseases

Dr.Kihito Takahashi, Japanese Association of Pharmaceutical 
Medicine (JAPHMED), Merck Banyu Pharma, Japan

\section{October 4, 2006 Wednesday}

0900-1030 Overview of chemistry in drug discovery

Hit/lead generation and optimisation

Dr. Prof. Yoshimoto, Nagasaki University,

Nagasaki, Japan

1030-1100 Tea break

1100-1200 Drug discovery for Prion disease

Prof. Shigeru Katamine, Nagasaki University, Nagasaki, Japan

1200-1300 Lunch

1300-1430 Visit laboratory in Nagasaki University (Prion Lab)

1430-1530 Drug discovery for TB

TBA

1530-1600 Tea break

1600-1700 Drug discovery for Trypanosomiasis

Prof. Dr. Kiyoshi Kita, University of Tokyo, Japan

\section{October 5, 2006 Thursday}

0900-1000 Publications, IPR and patents in drug discovery

Mr. Kenichi Osawa, Merck Banyu Pharma, Japan

1000-1030 Tea break

1030-1130 Publications, IPR and patents in drug discovery (Cont.)

Mr. Kenichi Osawa, Merck Banyu Pharma, Japan

\section{Chemical Manufacturing and Control (CMC)}

\section{October 6, 2006 Friday}

0900-1000 Synthesis of active pharmaceutical ingredient

Prof Susumi Hatakeyama, Nagasaki University, Japan

10:00-10:30 Formulation

Prof Susumi Hatakeyama, Nagasaki University, Japan

1030-1100 Tea break

1100-1300 Methods for determination of concentrations in various media by

means of spectrometric methods, HPLC, and biological methods

Prof.Dr. Masaaki Kai, Nagasaki University, Japan

1300-1400 Lunch

1400-1530 Stability for drug substance and drug prod- uct

Prof.Dr. Hiroaki Nagaoka, Nagasaki International University, Japan

1530-1600 Tea break

1600-1700 Development of specification

Prof.Dr. Hiroaki Nagaoka, Nagasaki International University, Japan

\section{October 7, 2006 Saturday}

0900-1030 Quality assurance/quality control

Prof.Dr. Hiroaki Nagaoka, Nagasaki International University, Japan

1030-1100 Tea break

1100-1200 Example: Antimalarial drug, dihydroartemisinin

Assoc. Prof. Supornchai, Mahidol University, Thailand

1200-1300 Lunch

1300-1530 Regulatory (with an example of a drug CMC requirement)

Prof.Dr. Hiroaki Nagaoka, Nagasaki International University, Japan

1530-1600 Tea break

1600-1630 Naming the New Chemical Entity (NCE)

Prof.Dr. Hiroaki Nagaoka, Nagasaki International University, Japan

\section{Pre-clinical Development}

Pharmacological development

9 October 2006 Monday

0900-1100 Pharmacological data in new drug application

Dr. Shunsuke Ono, University of Tokyo, Japan

1100-1130 Tea break

1130-1230 Methods in pharmacological R\&D (1)

Dr. Hiroyuki Itoh, Astellas Pharma Inc, Japan

1230-1330 Lunch

1330-1430 Methods in pharmacological R\&D (2)

Dr. Hiroyuki Itoh, Astellas Pharma Inc, Japan

1430-1500 Discussion

Drs. Shunsuke Ono and Hiroyuki Itou

1500-1530 Tea break

1530-1630 The cure oriented therapeutics for chronic renal failure with gene therapy

Dr. Tsutomu Kurosawa, Osaka University, Japan 


\section{Toxicology}

10 October 2006 Tuesday

0900-1000 Principles of toxicology

Assoc. Prof.Dr. Wongwiwat Tassaneeyakul,

Kon Kaen University, Thailand

1000-1100 Toxicological tests: in vitro \& in vivo: acute, subacute, chronic, special organ toxicology, reproduction toxicology, teratogenicity, mutagenicity, carcinogenicity studies

Assoc. Prof.Dr. Wongwat Tassaneeyakul, Kon Kaen University, Thailand

1100-1130 Tea break

1130-1300 Scheduling of toxicological studies in the development plan, the registration requirements, human \& animal pharmacology, the proposed clinical application and the forms of administration.

Dr. Soisanwan Satarug, University of Queensland, Australia

1300-1400 Lunch

1400-1530 Continuous monitoring of the correlation between new toxicological findings and the unwanted events observed in humans up till now.

Dr. Soisanwan Satarug, University of Queensland, Australia

1530-1600 Tea break

Pre-clinical Pharmacokinetics

11 October 2006 Wednesday

0900-1030 Principles of pharmacokinetics: ADME processes

Assoc. Prof.Dr. Wongwat Tassaneeyakul, Kon

Kaen University, Thailand

1030-1100 Tea break

1100-1230 Pharmacokinetic data analysis \& pharmacokinetic parameters

Assoc. Prof.Dr. Wongwat Tassaneeyakul, Kon Kaen University, Thailand

1230-1330 Lunch break

13:30-1530 Transferability of the pharmacokinetic findings in animals to humans investigating toxicological problems - practices and pitfalls

Dr. Soisanwan Satarug, University of Queensland, Australia

\section{October 2006 Thursday}

1000-1200 Visit animal facility for medical research (Sato animal house)

1500-1630 Evaluation of viability (risk and benefit) for further development
(Case study)

Dr Tadaaki Taniguchi, Japanese Association

of Pharmaceutical Medicine (JAPHMED),

Merck Banyu Pharma, Japan

\section{Clinical Development}

\section{Clinical Trial}

\section{October 2006 Friday}

0900-1100 Overview of clinical development

- Assessment of pre-clinical information

- Clinical development plan

- Application of pharmacokinetics and pharmacodynamics in drug development

Dose selection and regimen

Dr. Tadaaki Taniguchi, Japanese Association of Pharmaceutical Medicine (JAPHMED), Merck Banyu Pharma, Japan $n$

1100-1130 Tea break

1130-1200 The various investigational phases of clinical research (Phases I-IV)

Dr. Tadaaki Taniguchi, Japanese Association of Pharmaceutical Medicine (JAPHMED), Merck Banyu Pharma, Japan

1200-1300 Lunch

1300-1500 Human pharmacokinetics:

- Definition and significance of pharmacokinetic parameters ( absorption , bioavailability, binding to proteins, distribution, clearance, elimination half life, AUC)

- Special human-pharmacokinetic studies e. g. bioavailability studies of multiple-dose, interaction studies, pregnancy, liver disease etc .

Prof. Dr. Kesara Na Bangchang, Director, Graduate Program in Biomedical Sciences, Thammasat University, University, Thailand

$1500-1530$ Tea break

\section{October 2006 Saturday}

0900-1000 Therapeutic exploratory (with example)

Dr. Kenji Nonaka, Japanese Association of Pharmaceutical Medicine (JAPHMED), Merck Banyu Pharma, Japan

1000-1100 Therapeutic confirmatory (with example) Dr. Kenji Nonaka, Japanese Association of Pharmaceutical Medicine (JAPHMED), Merck Banyu Pharma, Japan Tea break

1100-1130 Tea Break

1130-1230 Therapeutic use (with example)

Dr.Kimihiro Kasamo, Japanese Association 
1230-1330

$1330-1500$

$1500-1530$

$1530-1630$

Study design

16 October 2006 Monday

0900-1030 Study design

- Possible study designs taking into account ethical aspects, indication, controls, patient population, location of the trial centers

- Trial design (parallel group design, cross over design, factorial design, group sequential design)

- Design techniques to avoid bias (blinding, randomization)

Prof. Dr. L. Jeeyaseelan, Christian Medical University, Vellor, India

1030-1100 Tea break

1100-1230 Study design (Cont.)

- Multi centers trials

- Type of comparison

- Outcome measurements

Prof .Dr. L. Jeeyaseelan, Christian Medical University, Vellor, India

1230-1330 Lunch

1330-1500 Statistical considerations

- Biostatistics in the planning phase (estimate of number of cases, randomization, statistical models, definition of end-points, planning of the subsequent evaluation)

- Statistical analysis plan

- Analysis sets: full analysis set, per protocol set, missing values and outliers
Prof. Dr. L. Jeeyaseelan, Christian Medical University, Vellor, India

1500-1530 Tea break

1530-1700 Statistical considerations (Cont.)

- Data transformation

- Method of statistical analysis (estimation, confidence intervals, hypothesis testing, evaluation of safety and tolerability)

- Statistical analysis report

Prof. Dr. L. Jeeyaseelan, Christian Medical University, Vellor, India

\section{Regulatory Issues}

17 October 2006 Tuesday

0900-1030 Regulatory aspects of clinical development Dr. Kazuhiko Mori, Office of New Drugl, Center for Product

Evaluation, Pharmaceutical and Medical Devices, PMDA, Japan

1030-1100 Tea break

1100-1230 Special topics:

- Genetic engineer product

- Gene therapy and stem cells

Dr. Kazuhiko Mori, Office of New Drug1, Center for Product

Evaluation, Pharmaceutical and Medical Devices, PMDA, Japan

1230-1330 Lunch

1530-1600 Example of Clinical Drug development in Inida - Miltefosine trial

Prof. Dr. Juntra Karbwang, WHO/TDR, Switzerland

\section{Traditional Medicine}

\section{October 2006 Wednesday}

0900-1030 Introduction to traditional Medicine: drug discovery and development

Professor Dr. Kiichiro Tsutani, University of Tokyo, Japan

1030-1100 Tea break

1100-1200 Guidance on herbal medicine

Prof. Dr. Juntra Karbwang, WHO/TDR, Switzerland

1300-1500 Regulation for traditional medicine development

Japan: Dr. Ichiro Arai, Manager, $R \& D$ Strategy Dept. Tsumura \& Co.

China: Dr. Luping Qin, China

Thailand: Dr. Vichai Chokevivat, Director, Department of Alternative Medicine, $\mathrm{MOH}$ Thailand 
1500-1530 Tea break

1530-1700 Example: Herbal medicine to modern medicine

Example: traditional medicine development Dr. Luping Qin, China

\section{Module 3: Vaccine Development Vaccine Discovery}

\section{October 2006 Thursday}

0900-0930 Historical of vaccine Discovery Dr. Howard Engers, AHARI, Ethiopia

0930-1030 Overview of modern vaccine discovery Dr. Howard Engers, AHARI, Ethiopia

1030-1100 Tea break

1100-1200 Screening for antigens

Prof Dr. Kenji Hirayama, Nagasaki University, Japan

1200-1330 Lunch

1330-1430 Evaluating antigens

Prof Dr. Kenji Hirayama, Nagasaki University, Japan

1430-1500 Tea break

1500- Visiting Vaccine Discovery Laboratory Institute of Tropical Medicine, Nagasaki University

\section{October 2006 Friday}

0900-1030 Adjuvant -

Dr. Howard Engers, AHARI, Ethiopia

1030-1100 Tea break

1100-1200 Alternatives to antigens: DNA vaccine, Live or attenuated pathogen

Dr. Howard Engers, AHARI, Ethiopia

1200-1330 Lunch

1330-1430 Selection of development candidate and back -ups

Dr. Howard Engers, AHARI, Ethiopia

1430-1500 Tea break

1500-1630 Efficacy, toxicity, route if immunization, price, stability, cold chain,

Dr. Howard Engers, AHARI, Ethiopia

\section{October 2006 Saturday}

0900-1030 Malaria vaccine discovery

Prof. Dr.Weiqing Pan, China

1030-1100 Tea break

1100-1200 Cholera vaccine discovery

Dr. Masahiko Ehara, Nagasaki University, Japan

1200-1330 Lunch
1300-1400 West Nile Fever vaccine discovery

Prof. Dr.Kouichi Morita, Nagasaki University, Japan

1400-1500 Oral vaccine discovery

Dr. Takeshi Arakawa, Ryukyu University, Japan

1500-1530 Tea break

\section{Antigen Development}

23 October 2006 Monday

0900-1030 Scale-up, manufacture and control:

Types of large scale production of antigen, GLP and GMP

Dr. Horiuchi, GlaxoSmithKline, Tokyo, Japan

1030-1100 Tea break

1130-1230 Synthesis of antigen

Peptide, recombinant protein, DNA vaccine, recombinant $\mathrm{BCG}$ or organism, live or attenuated organism

Dr. Horiuchi, GlaxoSmithKline, Tokyo, Japan

1230-1330 Lunch

1330-1430 Synthesis of adjuvant

Mixture type or recombinant type

Dr. Horiuchi, GlaxoSmithKline, Tokyo, Japan

1430-1500 Tea break

1500-1530 Formulation

Soluble or suspension, Route, frequency, interval, number of dose, with or without adjuvant, mucosal immunization (aerosol, oral, nasal, inhalation, food), instability

Dr. Horiuchi, GlaxoSmithKline, Tokyo, Japan

1530-1600 Quality assurance/quality control

Dr. Horiuchi, GlaxoSmithKline, Tokyo, Japan

\section{Clinical Development}

24 October 2006 Tuesday

0900-0930 The various investigational phases of clinical research (Phases I-IV)

Dr. Horiuchi, GlaxoSmithKline, Tokyo, Japan

0930-1030 Basic principles and evaluation of investigational results

- Phase-I and early Phase-II, with a view to further development

Dr. Horiuchi, GlaxoSmithKline, Tokyo, Japan 
1030-1100 Tea break

1100-1230 Basic principles for decisions regarding further development or discontinuation of a development project

Dr. Horiuchi, GlaxoSmithKline, Tokyo, Japan

\section{Pre-Clinical Development}

1330-1500 The use of humanized animal model

Dr. Kenji Hirayama, Nagasaki University, Japan

\section{October 2006 Wednesday}

0900-1030 Animal model used in pre-clinical studies Dr. Shigeyuki Kano, International Medical Center of Japan, Tokyo

1030-1100 Tea break

\section{Overview}

1100-1130 Assessment of pre-clinical information $T B A$

1130-1230 Clinical development plan Professor Dr. Kenji Hirayama, Nagasaki

1230-1330 Lunch

1330-1430 Application of immunogenicity for vaccine development

Dr. Shigeharu Ueda, The Research Foundation for Microbial Diseases of Osaka University (BIKEN), Japan

1430-1500 Tea break

1500-1600 Dose selection and regimen

Dr. Shigeharu Ueda, The Research Foundation for Microbial Diseases of Osaka University (BIKEN), Japan

\section{October 2006 Thursday}

\section{Pre-Clinical Development}

0900-1030 Safety assessment

Toxicity test for animal: regional complications, systemic toxicity such as fever, anaphylactic shock

Mr. Nobuhiro Noro, GlaxoSmithKline, Tokyo, Japan

1030-1100 Tea break

1100-1230 Immunogenicity assessment Mr. Nobuhiro Noro, GlaxoSmithKline, Tokyo, Japan

1230-1330 Lunch

1330-1430 Regulatory

Mr. Yoshino, Dr. Masaru Iwasaki, GlaxoS-
$1430-1500$

mithKline, Tokyo, Japan

1500-1600 Example: Malaria Vaccine Clinical Trial Development

TBA

1600-1700 Example: TB Vaccine Clinical Trial TBA

\section{Module 4: Diagnostic Development}

\section{October, 2006 Friday}

0900-1030 Discovery and development of diagnostic tools:

Necessity assessment, Principles and technology selection

Dr.Masato Sasaki, Roche Diagnostics KK, Tokyo, Japan

1030-1100 Tea break

1100-1230 Prototype production and assessment

Dr.Masato Sasaki, Roche Diagnostics KK, Tokyo, Japan

1230-1400 Lunch

1400-1530 Scale-up, manufacture and control

Dr. Masato Sasaki, Roche Diagnostics KK, Tokyo, Japan

1530-1600 Tea break

1600-1730 Scale-up, manufacture and control (Cont.)

28 October, 2006 Saturday

900-1030 Development of kits

Dr. Masato Sasaki, Roche Diagnostics KK, Tokyo, Japan

1030-1100 Tea break

1100-1230 Quality assurance/quality control: evaluation of efficacy after application

Dr. Masato Sasaki, Roche Diagnostics KK, Tokyo, Japan

1230-1400 Lunch

1400-1530 Clinical development: validate prototype, manufacture pilot lot, and initiate clinical trial

Dr. Masato Sasaki, Roche Diagnostics KK, Tokyo, Japan

1530-1600 Tea break

1600-1730 Clinical development: Supply chain logistics and production, Statistical consideration, regulatory issues

Dr. Masato Sasaki, Roche Diagnostics KK, Tokyo, Japan 
Module 5: Standards in Clinical Research and Development

Ethics in research and Ethics Committee

30 October, 2006 Monday

0900-1000 Ethics Codes and Guidelines

Prof. Dr. Cristina Torres, FERCAP, Thailand

1000-1100 Principles of Research Ethics

Prof. Dr. Cristina Torres, FERCAP, Thailand

1100-1130 Tea break

1130-1230 Case study

1230-1400 Lunch

1400-1500 Research methodology and ethical issues (1)

Traditional medicine

Dr. Vichai Chokevivat, Director, Department of Alternative Medicine, MOH Thailand

1500-1600 Research methodology and ethical issues (2) Genetic study

Prof. Dr. Kenji Hirayama, Nagasaki University, Japan

1600-1630 Tea break

1630-1730 Case study

\section{October, 2006 Tuesday}

0900-1030 Ethics Committee

Prof. Dr. Cristina Torres, FERCAP, Thailand

1030-1100 Tea break

1100-1230 Ethics committee Cont.

Prof. Dr. Cristina Torres, FERCAP, Thailand

1230-1400 Lunch

1400-1500 Data and Safety Monitoring Board (DSMB)

Prof. Dr. Juntra Karbwang, WHO/TDR, Switzerland

1500-1630 Case study

1630-1730 Monitoring and auditing Ethics Committee

Prof. Dr. Cristina Torres, FERCAP, Thailand

1 November, 2006 Wednesday

Quality Standards

0900-09.30 Concept of Good Clinical Practice

Dr. Johansen, Allan, Roche Products Pty limited, Australia

09.30-11.30 Responsibilities

Sponsor (Dr. Allan Johansen)

Investigators (Prof. Kenji Hirayama)

IRB (Prof. Cristina Torres)

Monitors (Prof. Juntra Karbwang)

DSMB (Dr. Allan Johansen)

11.30-12.00 Audit and Inspection

Dr. Johansen, Allan, Roche Products Pty limited, Australia

1200-1300 Lunch
14:00-15:30 New Asymmetric Catalysis; Leading to the synthesis of Tamiflu

Prof. Dr. Masakatsu Shibasaki, The University of Tokyo, Japan

2 November, 2006 Thursday

In the morning: Field Trip to Kaketsuken, Kumamoto by Bus:

13:00-17:00 Good Manufacturing Practice (GMP)

Good Laboratory Practice (GLP)

Dr. Kyousuke Mizuno, Kaketsuken, Kumamoto, Japan

Visit GMP lab and GLP lab and Plant for vaccine production

3 November, 2006 Friday Holiday

Module 6: Clinical Data Management

6 November, 2006 Wednesday

0900-1000 Overview of clinical data management

Data management plan

Dr. Charcrin Na-Bangchang, TU-CDMC, Thailand

1000-1030 Statistical Analysis Plan (SAP)

Data: primary \& secondary data

Dr. Rui Wang, SMMC-CDMC, China

1030-1100 Tea break

1100-1230 Data capture, development of database

Prof. Dr. L. Jeeyaseelan, CMC-CDMC, India

1230-1330 Lunch

1330-1400 Data entry, data verification, data validation, audit trail

Data clarification process

Data query and resolution

Prof. Dr. Kenji Hirayama, CMC-CDMC, Nagasaki University, Japan

Dr. Lawrence Yamua, AA-CDMC, Ethiopia

1400-1500 Data transform process

- Adverse Event Dictionary

- Drug Dictionary

Dr. Sangkae Chamnanawakit, TU-CDMC, Thailand

1500-1530 Tea break

1530-1700 Statistical analysis

Dr. Arunachalam Rajapopal, CMC-CDMC, India

1700-1800 Quality Control \& Assurance (QC \& QA)

Standard Operating Procedures (SOPs)

Dr. Jose Fernando Florez Arango, CMCCDMC, Colombia 
Module7: Post-registration Activities

\section{November 2006 Tuesday}

0900-1000 Stakeholders to be involved in making product development work for the intended beneficiaries

Prof. Dr. Chitr Sitthi-amorn, Chulalongkorn University, Thailand

Prof. Dr. Pakdee Pothisiri, FDA, Thailand

Prof. Dr. Kazuko Kimura, Kanazawa University, Japan

Dr.Kihito Takahashi, Japanese Association of Pharmaceutical

Medicine (JAPHMED), Merck Banyu

Pharma, Japan

1000-1100 Policy Instrument

Prof. Dr. Pakdee Pothisiri, FDA, Thailand

Prof. Dr. Chitr Sitthi-amorn, Chulalongkorn

University, Thailand

1100-1130 Tea break

1130-1230 Public private partnership

Prof. Dr. Chitr Sitthi-amorn, Chulalongkorn University, Thailand

Prof. Dr. Pakdee Pothisiri, FDA, Thailand

Prof. Dr. Kazuko Kimura, Kanazawa University, Japan

1230-1330 Lunch

1330-1430 Public private partnership Cont.

1430-1500 Tea break

1500-1700 Pharmacoeconomics
Prof. Dr. Kiichiro Tsutani, University of Tokyo, Japan

\section{November 2006 Wednesday}

0900-1700 Improving the quality of new products in health systems: International network of regional use of drugs

Prof. Dr. Chitr Sitthi-amorn (Chulalongkorn University)

0900-1030 Post-marketing product vigilance

Dr. Janis Lazdins, TDR/WHO, Geneva, Switzerland

Prof. Dr. Chitr Sitthi-amorn, Chulalongkorn University, Thailand

Prof. Dr. Pakdee Pothisiri, FDA, Thailand

1100-1130 Tea break

1100-1200 Capacities for optimal delivery of new products: training and health service research

Prof. Dr. Chitr Sitthi-amorn, Chulalongkorn University, Thailand

$1200-1300$

Lunch

1300-1430 Intellectual Property Rights Protection in Developing Countries

Prof. Dr. Hiroko Yamane, Graduate Institute for Policy Studies, Japan

1430-1500 Tea break

1500-1630 Product life cycle

Dr. Janis Lazdins, TDR/WHO, Geneva, Switzerland 